\title{
Contemporary rate of pacemaker implantation in patients discharged after TAVR
}

\author{
Nachiket Apte ${ }^{1}$, Sagar Ranka ${ }^{2}$, Moghni Mohammed ${ }^{2}$, Seth Sheldon ${ }^{2}$, Amit Noheria ${ }^{2}$, \\ Madhu Reddy ${ }^{3}$, Peter Tadros ${ }^{4}$, Sanjaya Gupta ${ }^{5}$, and Rhea Pimentel ${ }^{3}$ \\ ${ }^{1}$ University of Louisville \\ ${ }^{2}$ University of Kansas Department of Cardiovascular Medicine \\ ${ }^{3}$ University of Kansas \\ ${ }^{4}$ University of Kansas Medical Center \\ ${ }^{5}$ St. Luke's Hospital - Mid America Heart Institue
}

November 16, 2020

\begin{abstract}
Background: In-hospital permanent pacemaker implantation (PPMI) is a frequent and well-known complication of transcatheter aortic valve replacement (TAVR) procedure. The period of monitoring for need for pacing after discharge remains poorly understood. Methods: The National Readmission Database from first six months of calendar year 2016 and 2017 was queried for patient discharged alive after TAVR. All patients with prior pacemakers were excluded. Patients who received pacemaker after discharge (d-PPMI) were compared to a) patients receiving no pacemaker (o-PPMI) or b) patients receiving pacemaker on index admission(i-PPMI) over a 6 month follow-up. Results: Out of 39,993 patients who did not have a prior pacemaker, 4001(10.0\%) underwent PPM implantation during index admission (i-PPMI) while over the next 6 months, a further 734 (1.8\%) patients underwent the procedure (d-PPMI). For patients receiving PPMI during follow-up post TAVR discharge, the majority $(68 \%)$ occurred within 14 days. The primary cause of readmission for d-PPMI was heart block in majority of the cases $(73 \%$; complete heart block 49\%, second degree heart block 4\%, bradycardia/other heart block 20\%). The d-PPMI group also had a relatively shorter length of stay and a lower comorbidity burden when compared to the i-PPMI group. When compared to the o-PPMI group, the d-PPMI group were more likely to have higher advanced heart block. Conclusions: About one-fifth of pacemakers implanted post TAVR procedures happen during follow-up with a majority of them happening immediately after discharge. Risk stratification at discharge may help to identify patients who undergo PPMI post discharge.
\end{abstract}

Contemporary rate of pacemaker implantation in patients discharged after TAVR

Brief title: Post-TAVR rate of pacemaker implantation

Nachiket Apte $\mathrm{MD}^{1 *}$, Sagar Ranka $\mathrm{MD}^{2 *}$, Mohammed Moghni $\mathrm{MD}^{2}$, Seth Sheldon $\mathrm{MD}^{2}$, Amit Noheria MBBS SM${ }^{2}$, Madhu Reddy $\mathrm{MD}^{2}$, Peter Tadros $\mathrm{MD}^{2}$, Sanjaya Gupta $\mathrm{MD}^{3}$ \& Rhea Pimentel $\mathrm{MD}^{2}$

1: University of Louisville, Louisville KY

2: University of Kansas Health System, Kansas City KS

3: University of Missouri-Kansas City School of Medicine, Kansas City, MO

* These authors contributed equally and are co-first authors on this study

Total word count: Abstract - 268 words, Manuscript- 1929 words

Reprints and Correspondence: 
Rhea Pimentel, MD

Department of Cardiovascular Medicine

The University of Kansas School of Medicine

3901 Rainbow Blvd, Kansas City, KS 66160

Tel: $614-403-3890$

E-mail: rcpimentel@kumc.edu

\section{Structure Abstract:}

Background: In-hospital permanent pacemaker implantation (PPMI) is a frequent and well-known complication of transcatheter aortic valve replacement (TAVR) procedure. The period of monitoring for need for pacing after discharge remains poorly understood.

Methods: The National Readmission Database from first six months of calendar year 2016 and 2017 was queried for patient discharged alive after TAVR. All patients with prior pacemakers were excluded. Patients who received pacemaker after discharge (d-PPMI) were compared to a) patients receiving no pacemaker (o-PPMI) or b) patients receiving pacemaker on index admission(i-PPMI) over a 6 month follow-up.

Results: Out of 39,993 patients who did not have a prior pacemaker, 4001(10.0\%) underwent PPM implantation during index admission (i-PPMI) while over the next 6 months, a further 734 (1.8\%) patients underwent the procedure (d-PPMI). For patients receiving PPMI during follow-up post TAVR discharge, the majority (68\%) occurred within 14 days. The primary cause of readmission for d-PPMI was heart block in majority of the cases (73\%; complete heart block $49 \%$, second degree heart block $4 \%$, bradycardia/other heart block 20\%). The d-PPMI group also had a relatively shorter length of stay and a lower comorbidity burden when compared to the i-PPMI group. When compared to the o-PPMI group, the d-PPMI group were more likely to have higher advanced heart block.

Conclusions: About one-fifth of pacemakers implanted post TAVR procedures happen during follow-up with a majority of them happening immediately after discharge. Risk stratification at discharge may help to identify patients who undergo PPMI post discharge.

\section{INTRODUCTION}

Transcatheter aortic valve replacement (TAVR) is an increasingly popular alternative to surgical valve replacement and is indicated for a broad range of patients with aortic stenosis. In an analysis of the Thoracic Surgeons-American College of Cardiology Transcatheter Valve Therapy (TVT) Registry, there were more than 100,000 TAVR performed between 2015 to 2017 with an positive trend(1). Despite improvement in TAVR technology and device iterations, the need for subsequent pacemaker implantation post TAVR remains a common complication (2). The close relationship of the conduction system to the aortic annulus and subsequent injury with the prosthetic stent frame may result in high-grade or complete cessation of atria-ventricular (AV) conduction. Factors predicting downstream pacemaker implantation include both modifiable factors (type of valve, depth of implantation) as well as non-modifiable factors (increased age, presence of right bundle branch block, length of membranous septum) $(3,4)$. Furthermore, pacemaker implantation is associated with increased periprocedural morbidity and mortality $(2)$.

Though multiple studies have elucidated risk factors of periprocedural high grade AV block, the incidence and predictors of delayed AV conduction block remains poorly understood. New onset high grade conduction system disease has been noted in small studies opting for routine post-TAVR rhythm monitoring $(5,6)$. Delayed high grade AV block has been inconsistently defined as occurring sometime after valve implantation, although late bradyarrhythmias are not uncommon and remain clinically significant $(7,8)$. It remains unclear if delayed AV block is sporadic or occurs in a time-dependent manner after TAVR. Hence post-TAVR monitoring studies have chosen empirically defined time intervals to assess incidence of delayed AV block. 
(9-11) In this study, we used a nationwide registry to study the incidence and timing of post-discharge pacemaker impanation after index admission for TAVR.

\section{METHODS}

\section{Study design:}

This is a retrospective cohort study from the Nationwide Readmission Database (NRD), a part of the Healthcare Cost and Utilization Project (HCUP) maintained by the Agency for Healthcare Research and Quality. NRD contains de-identified patient level public discharge data from 22 states, with an average of 14 million discharges per year, accounting for $56.6 \%$ of US hospitalizations. The NRD data elements include hospital characteristics, patient demographics, chronic co-morbidities, procedures, primary and secondary discharge diagnoses, payment source, and total costs. Since the database contains de-identified data, this study was exempted from institutional review board approval.

\section{Study inclusion criteria and readmission analyses:}

Using NRD from first six months of 2016 and 2017 (January to June), we included adult patients (age [?]18 years) undergoing transcatheter aortic valve replacement using International Classification of Diseases, Tenth Edition, Clinical Modification and were discharged alive (See Supplementary table). We excluded patients with prior pacemaker status. The patients were followed up for total for 6 months after their index hospitalization and divided into two groups based on if they received a pacemaker during their follow up (Figure 1). For patients who had multiple admissions in the follow-up period, the first admission for pacemaker implantation post discharge was considered.

\section{Study Outcomes:}

The primary outcome of our study was to assess rate of new pacemaker implantation in patients discharged following TAVR. Secondary outcomes include:

1. Primary indication for pacemaker implantation resulting in readmission post TAVR.

2. Time from index admission for TAVR to readmission for pacemaker placement post discharge (d-PPMI)

3. Clinical factors differentiating patients who received delayed pacemaker implant post TAVR (d-PPMI) from patients with no pacemaker from discharge up to 6 months of follow-up (o-PPMI)

4. Clinical factors differentiating patients who received delayed pacemaker implant post TAVR (d-PPMI) from patients who underwent pacemaker implantation during index admission for TAVR (i-PPMI)

Statistical Analysis:

All analyses were performed on weighted data and were performed accounting for complex sampling design as recommended by HCUP. Categorical and continuous variables were reported as percentages and mean \pm standard error (SE), respectively. Dichotomous outcomes were reported as proportions and compared with using the Pearson $\chi^{2}$ test to evaluate for univariate associations. In-hospital mortality was modelled into a multivariate logistic regression model adjusting for demographics, co-morbidities, complications and treatment characteristics and reported as adjusted odds ratios (aOR) and their 95\% confidence intervals. All data extraction and analyses were performed using SPSS (Version 15; College Station, TX).

\section{RESULTS}

From 2016 to 2017, there were a total of 46077 patients who underwent TAVR implantation (Figure 1), out of which 6084 patients $(13.2 \%)$ with a prior pacemaker were excluded. Out of 39,993 patients who did not have a prior pacemaker, 4001 (10\%) underwent PPMI during index admission while 734 (1.8\%) underwent pacemaker implantation on readmission during their six-month follow up. Thus, the delayed pacemaker group (d-PPMI) represented $15.5 \%$ of all pacemakers implanted post TAVR implantation.

The mean time to pacemaker implantation post discharge for TAVR was 30.2 days ( \pm 46.9 days). There was a rapid increase in pacemaker implantation after the TAVR with $68 \%(\mathrm{n}=499)$ implanted within the first 14 days (Figure 2). Thereafter, the slope of the curve decreased around 20 days with a steady small rate 
of PPMI. In terms of causes for readmission (Figure 3), most were due to complete heart block (49\%) and other being- bradycardia/non-specific heart block 20\%, Second degree heart block (4\%), complications of prosthetic heart valve $(3 \%)$, tachyarrhythmias $(6 \%)$ and miscellaneous $(18 \%)$.

\section{Comparison of d-PPMI with i-PPMI}

Some notable differences between the d-PPMI and i-PPMI patients are worth mentioning. As seen in Table 1, the i-PPMI patients were older by approximately 1 year (81.1 years vs 79.9 years, $\mathrm{P}=0.01$ ), were more likely to have pulmonary vascular disease (such as pulmonary embolism), hypertension, ventricular tachyarrhythmias, uncomplicated diabetes, liver disease and coagulopathies. As seen in Table 2, the i-PPMI group had higher rates of advanced heart block $(80.1 \%$ vs $9.5 \% ; \mathrm{p}<0.001)$ and a longer mean length of stay ( 7.0 vs 3.7 days, $\mathrm{p}<0.001)$. In terms of other conduction system disease, the i-PPMI were more likely to have preexisting right bundle branch block(13.3\% vs $8.3 \%, \mathrm{p}=0.002)$, left bundle branch block ( $0.7 \%$ vs $0 \%$; $\mathrm{p}<0.001)$, non-specific heart block (1.4\% vs $0.17 \%$; $=<0.001)$ as well as bi-fascicular block $(7.7 \%$ vs $3.1 \%, \mathrm{p}<0.001)$.

Comparison of d-PPMI with o-PPMI

As seen in Table 1, the d-PPMI patients were less likely to be female ( $40.7 \%$ vs $46.9 \%$; $=0.04$ ). Interestingly, they had a significantly shorter length of stay for index hospitalization ( 3.7 days vs 4.9 days, $\mathrm{p}<0.001)$. They also had a higher incidence of atrial fibrillation, ventricular tachyarrhythmias, pulmonary vascular disease, uncomplicated diabetes. In comparing conduction system disorders (Table 2), the presence of advanced heart block was significantly higher in in d-PPMI $(10.8 \%$ vs $5.4 \% ; \mathrm{p}=0.001)$, with higher presence of preoperative right bundle branch block ( $8.3 \%$ vs $3.5 \%, \mathrm{p}=0.002)$, second degree heart block $(2.6 \%$ vs $0.8 \% ; \mathrm{p}=0.048)$ and bifascicular block(3.1\% vs $1.2 \% ; \mathrm{p}=0.02)$. First-degree heart block was higher in the d-PPMI group but was not statistically significant.

\section{DISCUSSION}

The current nationwide study evaluates the need for pacemaker placement during six-month follow up in patients after TAVR. The novel findings of this study that deserve emphasis are a) most pacemaker implantations occur within 14 days of discharge b) There is a sudden increase in pacemaker implantation after TAVR implantation that steadies at 20 days c) Most common primary reasons for readmission needing pacemaker implantation is complete heart block.

The STS TVT registry from 2011 to 2014 reported the median time to pacemaker implantation to be 3 days, and occurring often occurring at the time of index TAVR(12). This correlated with early experience of TAVR in the United States and first-generation devices. Our study selects a more contemporary cohort with inclusion of all-payer patients in the analysis. Our study also reflects patients who received TAVR valves after the FDA expanded the indication to intermediate risk patients that may reflect a lower burden of comorbidities. Our study had a nationwide sample with almost 2/3rds of patients getting PPMI at 14 days suggesting that it could be a reasonable timeframe to clinicians who can monitor at-risk patients for timely detection of need for permanent pacemakers though median time to PPMI was almost a month after TAVR implantation. It also has implications on discharge planning for patients who may be candidates for early discharge

The factors that adequately predict at-risk patients are multiple and currently non-standardized $(2,13,14)$. In a study of 611 patients, a higher prevalence of right bundle branch block and change in PR interval was predictive of delayed conduction disturbances.(15). Other factors like advanced age ( $>75$ years) and male gender have been shown to predict pacemaker implantation $(4,12)$. Certain risk scores that include pre-TAVR characteristics are tailored towards predicting periprocedural pacemaker implantations(16). Several studies have stressed the utility of pre- and post- procedural ECG changes to identify patients at risk of delayed pacemakers, their findings however have been inconsistent. $(7,15,17)$ A single center study even observed normal PR and QRS intervals at discharge .(6) The low incidence of pacemaker implantation after discharge may be a barrier to robust modelling and prediction. Even in our study, we found limited clinical differences in patients receiving a pacemaker post discharge compared to the patients who did not, suggesting a lack of 
clear predictor variables. Moreover, the lack of echocardiographic and procedural characteristics limits the prediction in our study.

Our study also evaluates the reasons for receiving pacemaker at readmission. Almost half of the patients underwent pacemaker implantation for complete heart block with a minority requiring it for other higher degree AV blocks. It could perhaps be that the patient's AV conduction system fails progressively in the unmonitored outpatient setting, until complete cessation of conduction. Other probable causes include sudden complete cessation of conduction down the AV node, without evidence of progression, either post procedural or during follow-up or intermittent but symptomatic advanced heart block. Factors like cuff swelling or progressive valve stent frame expansion that may account for early presentation of heart block but may not explain the presentation beyond 14 days. (6) In a cohort of 150 patients monitored for thirty days after TAVR, 12(8\%) developed delayed AV block with 75\% developing complete heart block and rest developing second degree AV block(10). The study also showed than up to $40 \%$ of patients were symptomatic. Thus, further studies are needed to evaluate for natural history of conduction system disease after TAVR.

The length of stay was longest in patients who received pacemaker during the index admission of all three groups in our study, as has previously been reported. Interestingly, hospital stay was shortest for patients who subsequently received a pacemaker on follow up. This may suggest an uneventful index TAVR perhaps due to a lower burden of comorbid conditions leading to early discharge. Additionally, $9.5 \%$ of patients had advanced AV block at index admission but underwent PPMI in follow-up. (Table 2). This may suggest failure to identify risk of incident conduction disease meriting PPMI in a shorter hospital observation period or presence of conduction disorders that may have been transient during TAVR implantation or have resolved prior to discharge. Newer expert opinion does provide a direction towards follow-up of these patients, however the optimal timing of discharge after TAVR remains open to debate.(8) If certain patients are selected for early discharge, the post-discharge risk of unplanned pacemaker implantation may be mitigated by continued short-term rhythm monitoring. Several single center studies have used wearable or implantable monitors (911), but protocolized follow up monitoring is needed for large scale implementation.

\section{Limitations:}

Multiple noteworthy limitations exist in our study. Due to inherent administrative nature of this database, coding errors could result in identification of comorbidities as well as procedures. The database lacks the procedural characteristics of the TAVR (type and size of valve) as well as the lack of post-procedural complications. The extent of baseline electrocardiographic findings (QRS duration, PR interval) is limited. Post-discharge follow-up and use of ambulatory monitoring remains unknown, which could influence the timing of pacemaker implantation. It is possible that some patients may have died unexpectedly during the initial months post TAVR before they could reach the hospital. This could underestimate the true incidence of conduction system abnormalities. We purposefully excluded defibrillator implantation as well as CRT implantation in both index as well as follow-up patients in order to avoid any confusion regarding indication for implantation in late follow-up. However, given these limitations, the large sample size of the NRD database makes it optimal for capturing the small but significant increase in pacemaker implantations after discharge.

\section{CONCLUSIONS:}

Of all pacemaker implanted within 6 months post TAVR, about $15.5 \%$ pacemakers are implanted in patients after discharge from a TAVR procedure. There is sharp increase in pacemaker implantations in patients discharged after a TAVR procedure, with two-thirds of procedures occurring withing the first 14 days after discharge. The rate of implantation seems to flatten at 3 weeks after discharge and a steady increase thereafter. Further studies in post-TAVR rhythm monitoring may be beneficial to identify these patients sooner and prevent associated morbidity.

\section{Authors' contributions}

NA and SR are joint first co-authors on the article. NA, SR and RP took part in the study concept and design; SR and MM was responsible for acquisition and statistical analysis of data, NA,SR,MM and RP took 
part in interpretation of data; NA,SR and RP drafted the manuscript, NA,SR,RP,MM , PT, MR AN,SS and SG were involved in the critical revision of the manuscript for important intellectual content; NA SR and $\mathrm{MM}$ and took part in the statistical analysis; NA,SR and RP were in charge of the study supervision.

\section{Declaration of conflicting interests}

The author(s) declared no potential conflicts of interest with respect to the research, authorship, and/or publication of this article.

\section{Funding}

The author(s) received no financial support for the research, authorship, and/or publication of this article.

\section{REFERENCES}

1. Vemulapalli S, Carroll JD, Mack MJ, Li Z, Dai D, Kosinski AS, et al. Procedural Volume and Outcomes for Transcatheter Aortic-Valve Replacement. 2019;380(26):2541-50.

2. Nazif TM, Dizon JM, Hahn RT, Xu K, Babaliaros V, Douglas PS, et al. Predictors and clinical outcomes of permanent pacemaker implantation after transcatheter aortic valve replacement: the PARTNER (Placement of AoRtic TraNscathetER Valves) trial and registry. (1876-7605 (Electronic)).

3. Maan A, Refaat MM, Heist EK, Passeri J, Inglessis I, Ptaszek L, et al. Incidence and Predictors of Pacemaker Implantation in Patients Undergoing Transcatheter Aortic Valve Replacement. Pacing and clinical electrophysiology : PACE. 2015;38(7):878-86.

4. Siontis GC, Jüni P, Pilgrim T, Stortecky S, Büllesfeld L, Meier B, et al. Predictors of permanent pacemaker implantation in patients with severe aortic stenosis undergoing TAVR: a meta-analysis. (1558-3597 (Electronic)).

5. Ream K, Sandhu A, Valle J, Weber R, Kaizer A, Wiktor DM, et al. Ambulatory Rhythm Monitoring to Detect Late High-Grade Atrioventricular Block Following Transcatheter Aortic Valve Replacement. 2019;73(20):2538-47.

6. Sandhu A, Tzou W, Ream K, Valle J, Tompkins C, Nguyen DT, et al. Heart Block After Discharge in Patients Undergoing TAVR With Latest-Generation Valves. 2018;71(5):577-8.

7. Chorianopoulos E, Krumsdorf U, Pleger ST, Katus HA, Bekeredjian R. Incidence of late occurring bradyarrhythmias after TAVI with the self-expanding CoreValve(ß) aortic bioprosthesis. Clin Res Cardiol. 2012;101(5):349-55.

8. Rodés-Cabau J, Ellenbogen KA, Krahn AD, Latib A, Mack M, Mittal S, et al. Management of Conduction Disturbances Associated With Transcatheter Aortic Valve Replacement. Journal of the American College of Cardiology. 2019;74(8):1086.

9. Tian Y, Padmanabhan D, McLeod Christopher J, Zhang P, Xiao P, Sandhu Gurpreet S, et al. Utility of 30-Day Continuous Ambulatory Monitoring to Identify Patients With Delayed Occurrence of Atrioventricular Block After Transcatheter Aortic Valve Replacement. Circulation: Cardiovascular Interventions. 2019;12(12):e007635.

10. Ream K, Sandhu A, Valle J, Weber R, Kaizer A, Wiktor DM, et al. Ambulatory Rhythm Monitoring to Detect Late High-Grade Atrioventricular Block Following Transcatheter Aortic Valve Replacement. Journal of the American College of Cardiology. 2019;73(20):2538.

11. Rodés-Cabau J, Urena M, Nombela-Franco L, Amat-Santos I, Kleiman N, Munoz-Garcia A, et al. Arrhythmic Burden as Determined by Ambulatory Continuous Cardiac Monitoring in Patients With New-Onset Persistent Left Bundle Branch Block Following Transcatheter Aortic Valve Replacement: The MARE Study. (1876-7605 (Electronic)). 
12. Fadahunsi OO, Olowoyeye A, Ukaigwe A, Li Z, Vora AN, Vemulapalli S, et al. Incidence, Predictors, and Outcomes of Permanent Pacemaker Implantation Following Transcatheter Aortic Valve Replacement: Analysis From the U.S. Society of Thoracic Surgeons/American College of Cardiology TVT Registry. JACC: Cardiovascular Interventions. 2016;9(21):2189-99.

13. Maan A, Refaat MM, Heist EK, Passeri J, Inglessis I, Ptaszek L, et al. Incidence and Predictors of Pacemaker Implantation in Patients Undergoing Transcatheter Aortic Valve Replacement. (1540-8159 (Electronic)).

14. Saint Croix GA-O, Lacy SA-O, Hrachian H, Beohar N. Clinical Impact of Preexisting Right Bundle Branch Block after Transcatheter Aortic Valve Replacement: A Systematic Review and Meta-Analysis. (1540-8183 (Electronic)).

15. Mangieri A, Lanzillo G, Bertoldi L, Jabbour RJ, Regazzoli D, Ancona MB, et al. Predictors of Advanced Conduction Disturbances Requiring a Late ([?]48 H) Permanent Pacemaker Following Transcatheter Aortic Valve Replacement. JACC: Cardiovascular Interventions. 2018;11(15):1519-26.

16. Kiani S, Kamioka N, Black GB, Lu MLR, Lisko JC, Rao B, et al. Development of a Risk Score to Predict New Pacemaker Implantation After Transcatheter Aortic Valve Replacement. 2019;12(21):2133-42.

17. Mazzella AJ, Sanders M, Vavalle J, Gehi A. EARLY VERSUS LATE PACEMAKER IMPLANTATION AFTER TRANSCATHETER AORTIC VALVE REPLACEMENT. Journal of the American College of Cardiology. 2020;75(11 Supplement 1):317.

TABLES AND FIGURES 


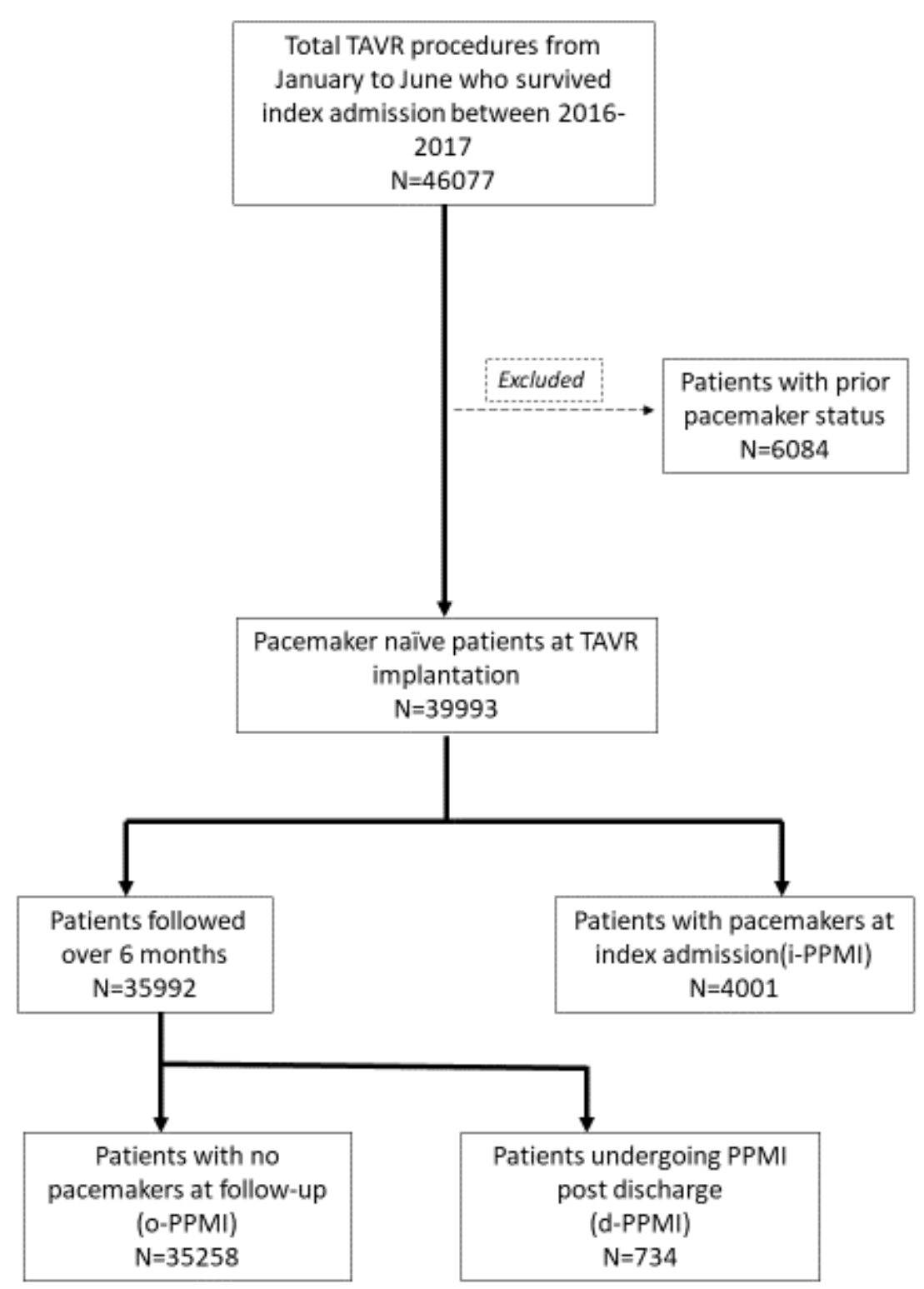

Figure 1: Algorithm showing selection of patients post TAVR admissions

\section{Hosted file}

image2 . emf available at https://authorea.com/users/360734/articles/493162-contemporary-rateof-pacemaker-implantation-in-patients-discharged-after-tavr

\section{Hosted file}

image3.emf available at https://authorea.com/users/360734/articles/493162-contemporary-rateof-pacemaker-implantation-in-patients-discharged-after-tavr 


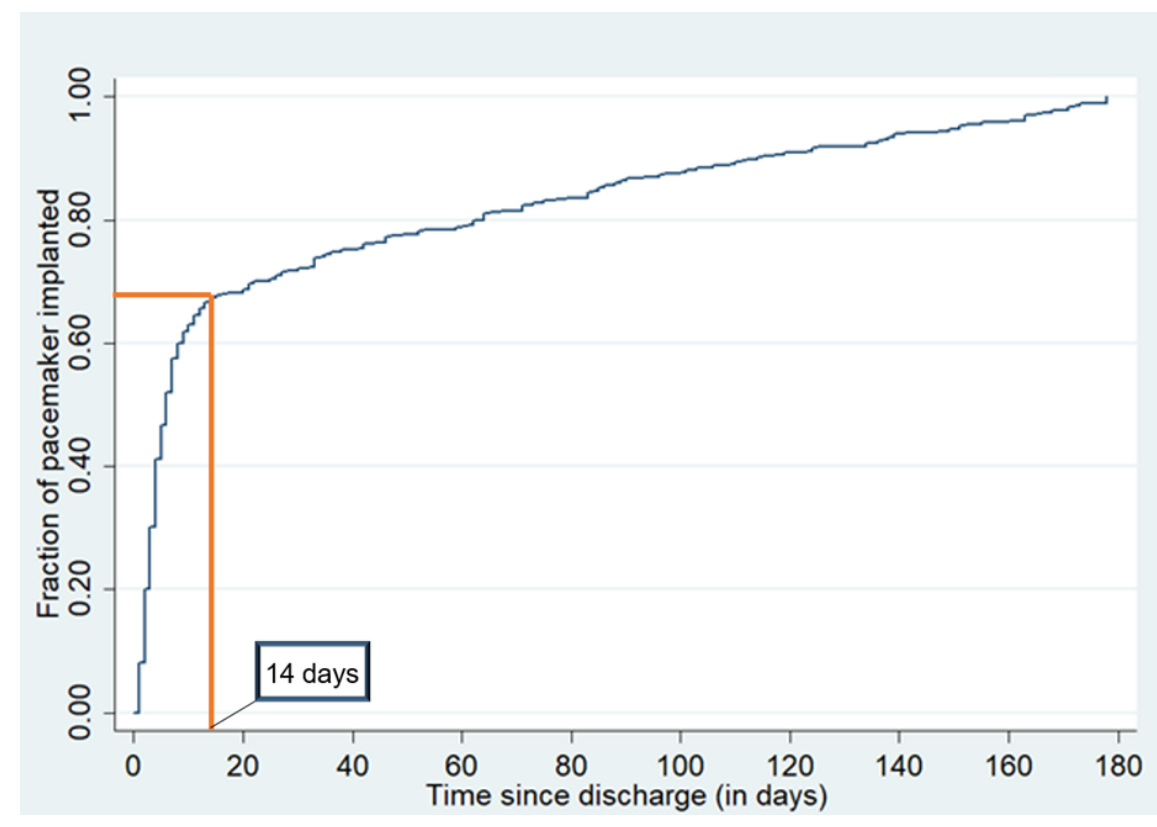

Figure 2: Rate of Delayed Pacemaker Implantation Post Discharge from TAVR Procedure

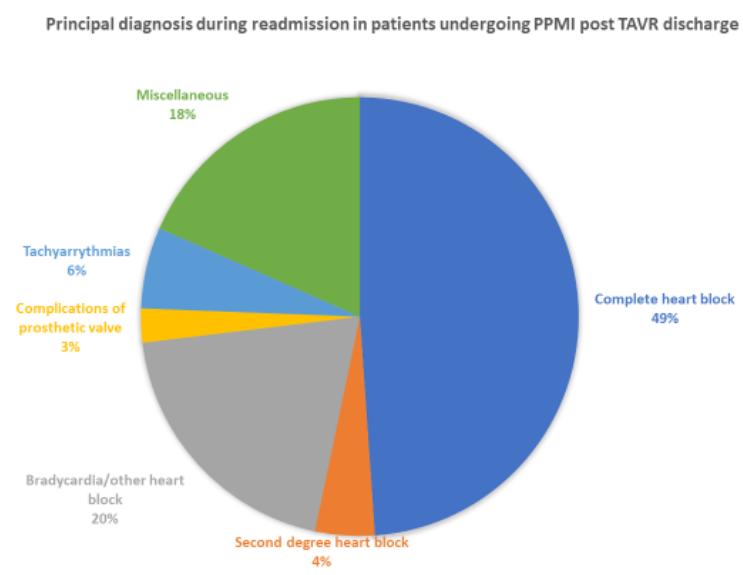

Figure 3: Pie chart indicating Principal Diagnosis for Readmission Post TAVR Implantation for patients who underwent pacemaker implantation post discharge over a 6 -month follow-up.

\section{SUPPLEMENTARY}

Table 1. List of the Key International Classification of Diseases, 9th \& 10th Revisions, Clinical Modification Codes Used in the Study.

Transcatheter Aortic Valve Replacement ICD-10-CM 02RF3JZ, 02RF3KZ, 02RF38Z, 02RF37Z, 02RF3JZ, 02RF37H, 02RF38H, 02RF3JH, 02RF3KH

History of Prior Pacemaker/ICD: Prior Pacemaker

ICD-10-CM Z950, Z450, Z4501, Z45018, Z4502, Z95810 
History of Conduction Disorder:

ICD-10-CM I444, I445, I4460, I4469, I447, I450, I4510, I4519, I452, I453, I454, I4589, I459

New Permanent Pacemaker Implantation:

ICD-10-CM 0JH604Z，0JH605Z，0JH606Z，0JH634Z，0JH635Z，0JH636Z，0JH806Z，0JH836Z，0JH804Z， 0JH834Z, 0JH805Z

, $0 \mathrm{JH} 835 \mathrm{Z}$

\section{Hosted file}

image6.emf available at https://authorea.com/users/360734/articles/493162-contemporary-rateof-pacemaker-implantation-in-patients-discharged-after-tavr 